\title{
Endoscopic removal of an impacted bile duct self-expanding metal stent (SEMS) using the SEMS-in-SEMS technique
}

In contrast to covered self-expanding metal stents (c-SEMS), retrieval of uncovered SEMS (u-SEMS) from the bile duct may be quite difficult or impossible. We describe the endoscopic removal of an impacted u-SEMS using the SEMS-in-SEMS technique.

A 62-year-old man presented with acute pancreatitis, jaundice, and weight loss. Computed tomography (CT) revealed a "malignant-appearing" mass in the head of the pancreas causing bile duct dilation. The patient underwent placement of a u-SEMS to palliate the jaundice and as a bridge to surgery. Upon referral, endoscopic ultrasound was performed with fine-needle aspiration of the pancreatic head lesion. Cytology was negative for malignancy. In addition, follow-up CT 4 weeks later revealed resolution of the mass.

Over a period of 9 weeks, the clinical status of the patient remained stable, without weight loss or B symptoms. Removal of the SEMS was therefore planned. Despite using multiple utensils, including oval and hexagonal snares, rat tooth and alligator forceps, and changes in scope position and torquing maneuvers, it was impossible to remove the u-SEMS during three different ERCP sessions. During the third ERCP, a decision was made to place a c-SEMS inside the impacted u-SEMS (๑ Fig. 1).

The patient returned 10 days later for stent extraction. During ERCP, a snare was placed tightly around both stents, but removal was still impossible as the snare slipped off repeatedly. Therefore, a rat tooth forceps was used to grab the inner stent ( Video 1 ). Constant traction was applied by pulling and torquing the scope for about 2-3 minutes. Both stents were retrieved completely ( $\bullet$ Fig. 2; $\bullet$ Video 1 ). Cholangiogram did not reveal any bile duct damage. The patient was doing well 1 month later.

The stent-in-stent technique has been reported to be useful for the removal of impacted SEMS in the esophagus [1]. Our report is of interest because it demonstrates that this salvage technique may be applicable to impacted u-SEMS in the bile duct. The mechanism may be similar to the

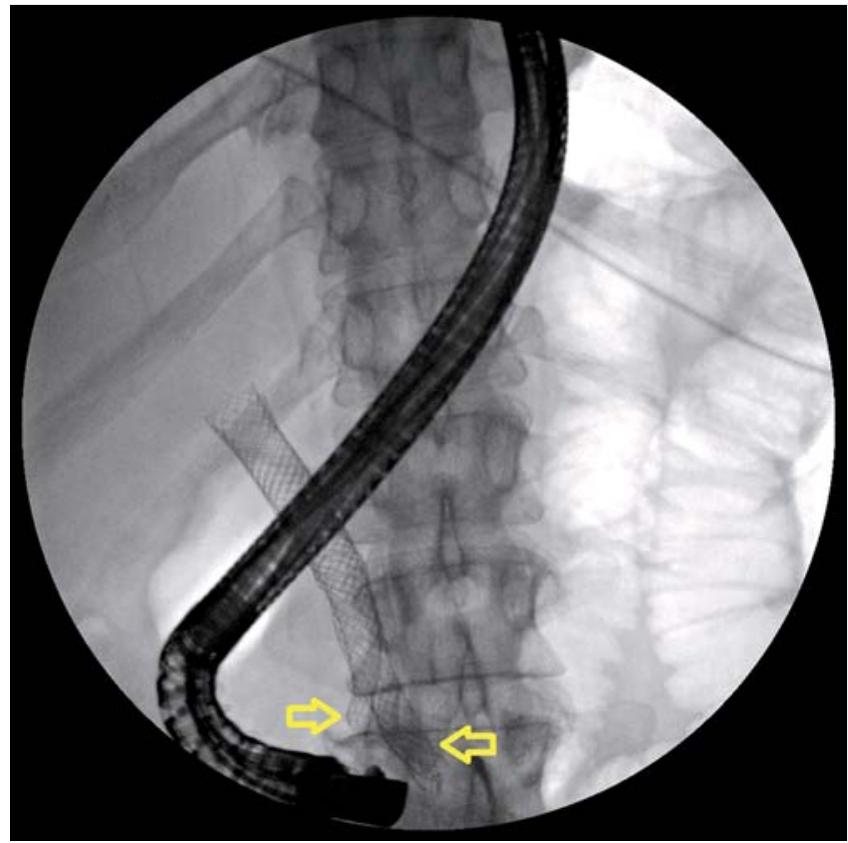

Fig. 1 Radiograph showing the fully covered self-expanding metal stent within the uncovered metal stent.

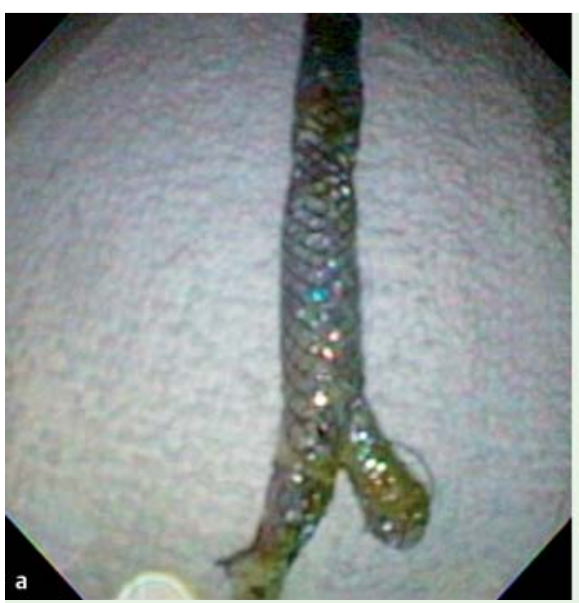

Fig. 2 Both self-expanding metal stents (SEMS) could be retrieved completely. a The covered SEMS was inserted through the side mesh of the

one described for impacted esophageal stents [1]: the newly inserted c-SEMS exerts radial pressure inside the previous u-SEMS leading to sloughing or necrosis of the inner lining of the bile duct. Our report is also of potential clinical interest as there is a modern trend to use SEMS for benign pancreatobiliary disease. Knowledge of stent removal techniques is therefore mandatory. Reported techniques to remove SEMS include snare, rat tooth forceps, wire cutter, invagination and extrac-

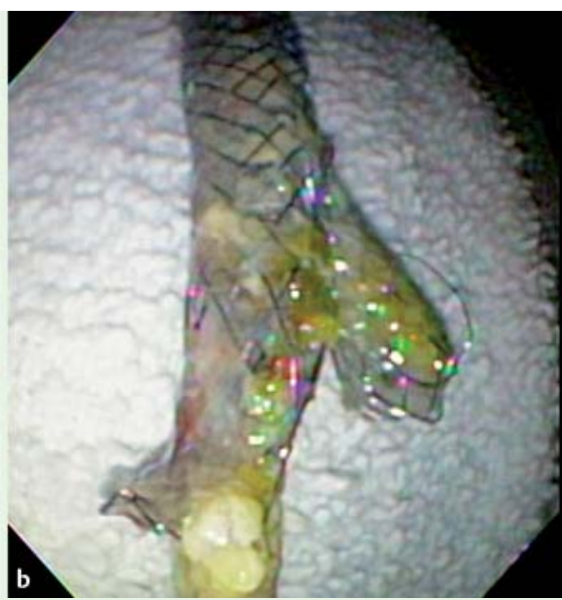

uncovered stent; $\mathbf{b}$ Close-up of both covered and uncovered SEMS.

\section{Video 1}

Rat tooth forceps was used to grab the uncovered self-expanding metal stent and then constant traction was applied by pulling and torquing both the rat tooth forceps and the scope for about 2 minutes. Under fluoroscopic view, careful attention was paid to observe stent dislodgement and its movement through the luminal upper gastrointestinal tract. Once retrieved through the mouth, the stents were placed on the table and examined for completeness of extraction. 
tion in pieces [2,3]. Our technique of intact snare retrieval using the SEMS-in-SEMS method may therefore be added to the armamentarium of the interventional pancreatobiliary endoscopist.

Endoscopy_UCTN_Code_TTT_1AR_2AZ

Competing interests: None
N. González ${ }^{1,2}$, J. Ramesh¹,

\section{M. Wilcox ${ }^{1}$, K. Mönkemüller ${ }^{1}$}

${ }^{1}$ Division of Gastroenterology and Hepatology, Basil Hirschowitz Endoscopic Center of Excellence, University of Alabama, Birmingham, Alabama, USA

2 Department of Gastroenterology, Hospital de Clínicas, Montevideo, Uruguay

\section{References}

1 Hirdes MM, Siersema PD, Houben MH et al. Stent-in-stent technique for removal of embedded esophageal self-expanding metal stents. Am J Gastroenterol 2011; 106: 286 293

2 Kahaleh M, Tokar J, Le T et al. Removal of selfexpandable metallic Wallstents. Gastrointest Endosc 2004; 60: 640 - 644

3 Egan LJ, Baron TH. Endoscopic removal of an embedded biliary Wallstent by piecemeal extraction. Endoscopy 2000; 32: $492-494$
Bibliography

DOI http://dx.doi.org/ 10.1055/s-0033-1344414

Endoscopy 2013; 45: E254-E255

(c) Georg Thieme Verlag KG

Stuttgart · New York

ISSN 0013-726X

Corresponding author K. Mönkemüller, MD, PhD, FASGE

Division of Gastroenterology and Hepatology Basil Hirschowitz Endoscopic Center of Excellence Endoscopy Unit, JT 664

619 19th Street $S$

Birmingham, AL 35249

USA

Fax: +1-205-934-1537

klaus1@uab.edu 